\title{
CART cell therapy for prostate cancer: status and promise
}

This article was published in the following Dove Medical Press journal: OncoTargets and Therapy

\author{
Haiyuan $\mathrm{Yu}^{1}{ }^{1, *}$ \\ Jun $\operatorname{Pan}^{1, *}$ \\ Zhicheng Guo' \\ Chunhua Yang ${ }^{1,2}$ \\ Lijun Mao ${ }^{1,2}$ \\ 'Department of Urinary Surgery, the \\ Affiliated Hospital of Xuzhou Medical \\ University, Xuzhou 221000, China; \\ ${ }^{2}$ Jiangsu Key Laboratory of Biological \\ Cancer Therapy, Department of Clinic \\ Institute, Xuzhou Medical University, \\ Xuzhou 22I002, China \\ *These authors contributed equally \\ to this work
}

Correspondence: Lijun Mao Jiangsu Key Laboratory of Biological Cancer Therapy, Xuzhou Medical University, No 99, Huaihai West Rd, Xuzhou, Jiangsu 221000, China

Tel +865168560 9999

Email maolijunxz@I63.com

Chunhua Yang

Department of Urinary Surgery, the Affiliated Hospital of Xuzhou Medical University, No 99, Huaihai West Rd,

Xuzhou, Jiangsu 221000, China

Tel +8651685609999

Email yangchunhua820203@I26.com

\begin{abstract}
In recent years, the, chimeric antigen receptor $\mathrm{T}$ (CAR-T) cell therapy as an adoptive immunotherapy has received great attention and made great breakthroughs. CAR-T cells show great specificity, targeting, and less major histocompatibility complex restriction in tumor immunotherapy, significantly different from traditional T cells. In spite of the progress of CART-T technology in the treatment of lymphoma, leukemia, and other blood system tumor, there are still many difficulties in the treatment of solid tumors by CAR-T technology. In this review, we will make a brief summary of the present situation of CAR-T cells in the treatment of prostate cancer, and discuss the promise of the application of this technology to prostate cancer therapy.
\end{abstract}

Keywords: prostate cancer, CAR-T cells, adoptive immunotherapy, review

\section{Introduction}

With the improvement of human living standard and the increase of life expectancy, the incidence of prostate cancer in the middle-aged and elderly males is increasing. Prostate cancer has become the most common tumor among males in Europe and the USA. ${ }^{1}$ Due to the lack of full understanding of the pathogenesis of prostate cancer, most patients are in the middle and late stage when the tumor is diagnosed and miss the best time for surgical therapy. Consequently, prostate cancer has high mortality. Androgen deprivation therapy is effective for the treatment of early stage prostate cancer because of the characteristics of androgen dependence in early development of prostate cancer. However, after androgen deprivation therapy, most of the patients develop castration-resistant prostate cancer (CRPC), which will finally develop into the metastatic castration-resistant prostate cancer (mCRPC). ${ }^{2}$ The development of mCRPC may be related to androgen receptor gene amplification, and regulatory factors of androgen receptors are abnormally expressed in prostate cancer. ${ }^{3}$ There is still no effective treatment for patients with CRPC. ${ }^{4}$

The chimeric antigen receptor $\mathrm{T}$ cell (CAR-T) therapy has gained great attention due to the advantages of CAR-T cells in the treatment of malignant tumors of the blood system and has achieved great breakthroughs. ${ }^{5,6} \mathrm{CAR}-\mathrm{T}$ technology provides a new way for the treatment of malignant solid tumors including prostate cancer. Up to now, there are only two ongoing clinical trials for prostate cancer CAR-T therapy. One is a Phase I trial of prostate-specific membrane antigen (PSMA)targeted CAR-T in mCRPC patients (NCT01140373). Another (NCT03089203) is a Phase I trial of "CART-PSMA-TGFßRDN Cells for CRPC" to evaluate the safety and feasibility of intravenously administered, lentivirally transduced, dual PSMA-specific/TGF $\beta$-resistant, CAR-modified autologous T cells (CART-PSMA- 
TGF $\beta$ RDN cells) in mCRPC patients. ${ }^{7}$ However, there are still many difficulties in the treatment of solid tumors by CAR-T technology. In this review, we make a systematic summary of the present situation of CAR-T cells in the treatment of prostate cancer and discuss the promise of the application of this technology to prostate cancer therapy.

\section{The molecular structure of CAR-T}

The CARs are fusion proteins constructed by modern molecular biotechnology. CARs are generally composed of three parts: extracellular antigen identification zone, transmembrane zone, and intracellular signal transduction zone. ${ }^{5}$ The extracellular antigen identification zone is the basis of specific recognition of tumor antigens by CARs. ${ }^{6}$ Single-chain fragment variable ( $\mathrm{scFv}$ ) of CAR-T cells can recognize tumor-associated antigen (TAA) specifically. The transmembrane zone usually consists of the transmembrane region of $\mathrm{CD} 3, \mathrm{CD} 8, \mathrm{CD} 28$, or FceRI and can fix scFv on the surface of $\mathrm{T}$ cells and transduce the signal into the cells. The intracellular signal transduction zone is composed of $\mathrm{CD} 8, \mathrm{CD} 28$, or CD137 intracellular area and $\mathrm{CD} 3 \zeta$, which contain the immune-receptor tyrosine-based activation motif (ITAM). ITAM plays a crucial role in the transduction of signal to active $\mathrm{T}$ cells.

\section{Antigen recognition}

$\mathrm{T}$ cell-mediated immune response is cellular immunity. According to the source of antigen, the pathway of activating $\mathrm{T}$ cells is different. The antigen presenting cell (APC) takes in the exogenous antigens and disassembles them into peptides by lysosomal degradation. Those peptides can combine to a major histocompatibility complex II (MHC II) to form MHC II-Ag complexes that can be recognized by $\mathrm{CD} 4^{+} \mathrm{T}$ cells. The endogenous antigens derived from cancer or virus-infected cells are absorbed by APC and disassembled into peptides by cytoplasmic protease system, which can combine with MHC I to form MHC I-Ag complexes that can be recognized by $\mathrm{CD} 8^{+} \mathrm{T}$ cells. ${ }^{8}$

\section{The activation and proliferation of $\mathrm{T}$ cells}

The complete activation of $\mathrm{T}$ cells requires the help of dual signal systems and cytokines. First signal comes from the combination of TCR and MHC I-Ag and the transduction of specific antigen stimulation signals into the cells by CD3. The signal can and enhance the affinity of LFA-1 with inter-cellular adhesion molecule (ICAM), which plays an important role in prolonging and stabilizing the combination of TCR and MHC I-Ag and the activation of T cells. The second signal is produced by the interaction between $\mathrm{T}$ cell membrane and APC membrane surface, mainly between B7 (CD80, CD86) on APC membrane and CD28 on T cell membrane. The double signals activate different signaling pathways and a variety of transcription factors to promote the production of cytokines such as IL-2 that can active T cells fully and make them survive longer. ${ }^{9}$ The activated T lymphocytes proliferate rapidly, and $\mathrm{CD} 8^{+} \mathrm{T}$ lymphocytes further differentiate into cytotoxic $\mathrm{T}$ lymphocytes that migrate to specific antigen aggregation site for biological effects.

\section{CAR-T}

With the development of genetic engineering technology, CARs could be designed and transfected into $\mathrm{T}$ lymphocytes of patients by transfection in vitro. Currently, in vitro transfection technology can be divided into viral transduction and nonviral transfection, the former includes retrovirus and slow virus, whereas the latter includes transposon system and mRNA electrotransfection.

CAR-T cells can be divided into four generations according to the costimulating molecules (CM; Figure 1). The first generation of CAR-T have no CMs and only contain a group of intracellular signals $(\mathrm{TCR} / \mathrm{CD} 3 \zeta$ chain or FceRI $\gamma$ chain) that can be combined with specific antigen

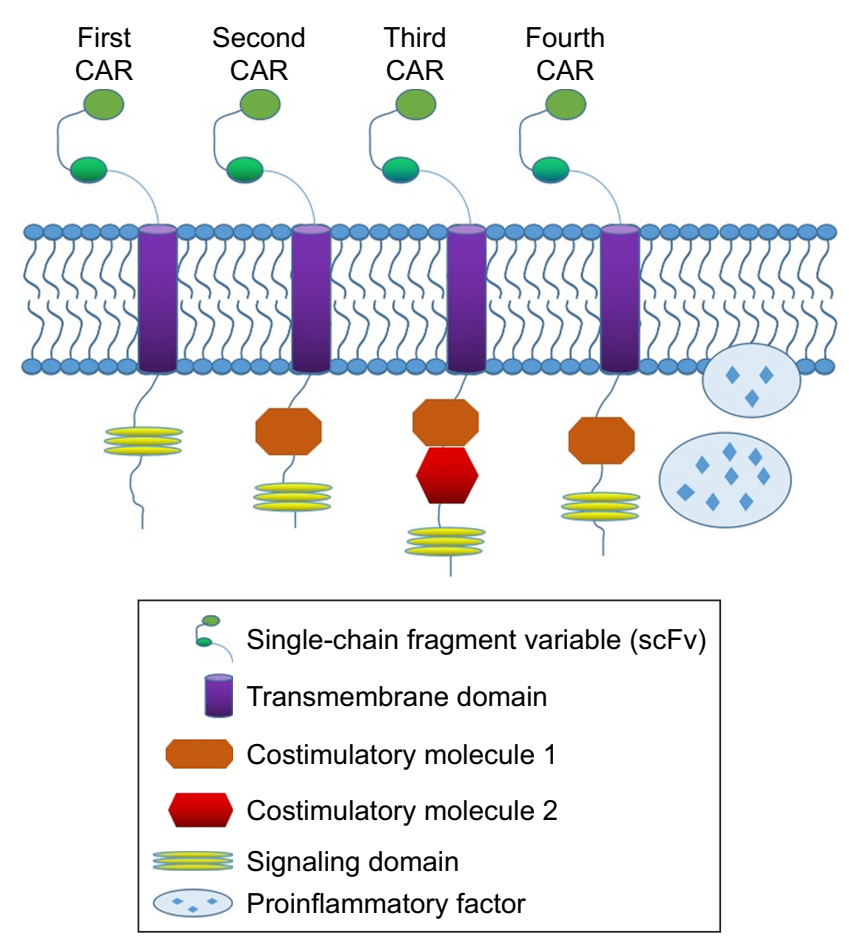

Figure I Diagram of the structures of CARs.

Note: Typical domain structures of the first to the fourth generation of CARs are shown.

Abbreviation: CAR, chimeric antigen receptor. 
and activate $\mathrm{T}$ lymphocytes. However, due to the lack of assistance of costimulation molecules, CAR-T cells transfused into patients have poor proliferation and cannot get expected effects. ${ }^{10}$ For the second generation of CAR-T, a costimulatory molecule such as CD28, CD27, 41BB (CDB7), OX40 (CD134), or inducible costimulatory molecule is added into $\mathrm{T}$ cells to overcome the failure of the first generation of CAR-T. Therefore, the ability of the second generation CAR-T cells to kill tumor cells is significantly improved. For the third generation of CAR-T, another CM such as $\mathrm{CD} 28,4-1 \mathrm{BB}$, or $\mathrm{CD} 3 \zeta$ is added into $\mathrm{T}$ cells to contain two stimulating molecules. Consequently, $\mathrm{T}$ cell proliferation activity and cytotoxicity as well as T cell survival are further improved. ${ }^{11}$ The fourth generation of CAR is called TRUCKS (fourth-generation CAR T-cells redirected for universal cytokine killing), which is different from the previous CAR-T on the structure. The addition of a proinflammatory factor such as IL12 and co-stimulatory molecule ligands (4-1 BBL and CD40L) provides CAR-T cells with higher efficacy of killing tumor cells through the release of a proinflammatory factor. ${ }^{12}$ In addition, patients who adopt the therapy with the fourth generation CAR-T cells can avoid pretreatment such as general or high-dose chemotherapy.

\section{Prostate-associated antigens}

\section{Prostate-specific antigen}

Currently, prostate-specific antigen (PSA) is the most common biomarker for prostate cancer. As a glycoprotein composed of 343 amino acids containing sugar chain, PSA is secreted by prostate gland vesicle and catheter epithelial cells with the activity of neutral serine protease and can be detected in the tissue, serum, and sperm. Because PSA has prostate tissue specificity, PSA is a good target to deliver drugs to tumor tissue to treat prostate cancer. However, due to serum PSA, avoiding the neutralization of targeted drugs and the side effects by serum PSA is still a difficult problem. Recent studies show that free prostate-specific antigen (fPSA) three hypotypes, namely benign prostate-specific antigen, inactive prostate-specific antigen, and precursor of PSA (pro-PSA). Among the three hypotypes of fPSA, pro-PSA has a higher expression in prostate cancer cells compared to the other two hypotypes, which makes it a more appropriate target for prostate cancer therapy. ${ }^{13}$

\section{Prostate acid phosphatase}

The prostate acid phosphatase (PAP) is another tumor marker for prostate cancer. Compared with PSA, PAP can accurately indicate the micrometastasis of prostate cancer and has been used as an immunotherapy target for CRPC. ${ }^{14,15}$ PAP is expressed not only in the tissues of the prostate and prostate cancer but also in small bowel cancer, pancreatic endocrine tumor, and bladder cancer. ${ }^{16}$ Similar to PSA, PAP serum level is extremely low under normal circumstances. Only after the prostate tissue is damaged, such as prostate cancer, a large amount of PAP will be released into the blood, which causes similar difficulty in the treatment of prostate cancer.

\section{Prostate-specific membrane antigen}

PSMA is a type II transmembrane glycoprotein expressed on prostate epithelial cell membrane. PSMA expression is low in ovarian and breast cell membranes and almost absent in the intestines, liver, kidney cell membranes, but is high in prostate tissue and vessels of solid tumors, with the highest PSMA expression in semen. ${ }^{15}$ Thus, PSMA has a good prostate tissue specificity and is the basis for PSMA targeted therapy of prostate cancer. Furthermore, PSMA may function as a receptor to engage signaling to promote cell migration and regulate the stability of chromosomes. ${ }^{16}$ Therefore, PSMA is a promising target for the treatment of prostate cancer.

\section{Prostate stem cell antigen (PSCA)}

PSCA is a TAA identified in prostate cancer cells. ${ }^{17}$ PSCA consists of highly conserved cysteine residues, signal sequence in the $\mathrm{N}$ terminal, a number of glycosylation sites, and $\mathrm{C}$ terminal glucose phosphatidyl inositol anchor sequence by which PSCA is fixed on the cell membrane of prostate cells. PSCA can be detected only on cell membrane since this antigen is not released into the blood. The expression rate of PSCA in prostate cancer tissue is almost $90 \%$, much higher than that in normal prostate tissue. With the progression of prostate cancer, PSCA expression level in cancerous tissues gradually increases and even reaches as high as 100\% in prostate cancer bone metastases. The expression level of PSCA is positively correlated with tumor grade and clinical stage. ${ }^{18}$ Furthermore, the expression of PSCA has not been detected in the testis, spleen, liver, bone marrow, lymph nodes, lung, bladder, kidney, cerebellum, and colon tissue. ${ }^{19}$ Because of prostate tissue specificity, no release to the blood, and high expression in prostate cancer tissues, PSCA serves as an important target for prostate cancer targeted therapy.

\section{CAR-T therapy in prostate cancer}

$\mathrm{Ma}$ et al made the second-generation anti-PSMA CAR-T cells by embedding CD28 as a costimulator. ${ }^{20}$ They used the healthy donor $\mathrm{T}$ cells to make the first-generation anti-PSMA 
IgTCR CAR-T cells and the second-generation anti-PSMA IgCD28TCR CAR-T cells with retrovirus. Compared with mice inoculated with nontransduced $\mathrm{T}$ cells, tumor volume in mice inoculated with anti-PSMA IgTCR-transduced $\mathrm{T}$ cells reduced significantly. However, in mice inoculated with anti-PSMA IgCD28TCR-transduced T cells, tumor volume reduced further significantly and the tumor nearly disappeared after 3 weeks. CAR-T cells targeted at PSMA showed potent killing effect against prostate cancer, and the second-generation CAR-T cells have a better killing effect than the first generation and provide a novel approach for clinical immune-targeted therapy for CRPC.

It is important to note that healthy tissues that express the targeted antigen may subject to $T$ cell-mediated damage. To solve this problem, Kloss et al came up with a strategy that $\mathrm{T}$ cells were transduced with both a CAR that provided suboptimal activation upon binding of one antigen and a chimeric costimulatory receptor that recognized a second antigen. ${ }^{21}$ Their results showed that cotransduced $\mathrm{T}$ cells destroyed only the tumors expressing both antigens but did not affect tumors expressing either antigen alone. This "tumor-sensing" strategy could avoid some side effects of targeted T-cell therapies and advance the application of CAR-T cell therapies.

\section{Conclusion}

CAR-T cells targeting prostate cancer-specific antigens have shown exciting results in in vitro cell and animal model experiments, but their efficacy and safety in clinical trials need further studies. For example, a recent study demonstrated the importance of costimulation in defining an optimal CAR-T therapy for PSCA+metastatic prostate cancer. ${ }^{22}$ Furthermore, Zhang et al recently developed CAR-T cells carrying an anti-PSMA chimeric TCR and a dominant negative TGFß type II receptor for the resistance to TGFßmediated suppression of cytotoxic $T$ cell function. The cells also expressed HSV1 thymidine kinase to enable cell killing via ganciclovir treatment. ${ }^{23}$ This novel strategy will overcome the immunosuppression problem frequently associated with cancer immunotherapy. Moreover, nanoparticle-mediated gene delivery has been shown to provide promising results for prostate cancer treatment. ${ }^{24}$ These results encourage the development of nanoparticles for the delivery of CAR-T in prostate cancer therapy. ${ }^{25}$ However, there are still many unknown questions in the field such as CAR-T-related signaling pathways, and the comparison with different generations of CAR-T cells still needs clinical trials to test. In addition, the role of stem cells in CAR-T therapy remains unclear. ${ }^{26}$ Future directions in the development of
CAR-T for prostate cancer treatment should focus on high specificity and efficacy, low toxicity and immunosuppression, and synergy with other tumor therapies. ${ }^{27}$ With the advances in new techniques such as clustered regularly interspaced short palindromic repeats (CRISPR), we expect that CAR-T technology can be successfully applied in clinical prostate cancer therapy in the near future.

\section{Acknowledgment}

This study was supported by grants from Jiangsu Province Science Foundation of China (No BK20151166), the Project of Invigorating Health Care Through Science, Technology and Education (No CXTDA2017034), and Jiangsu Provincial Medical Youth Talent (No QNRC2016794).

\section{Disclosure}

The authors report no conflicts of interest in this work.

\section{References}

1. Santoro SP, Kim S, Motz GT, et al. T cells bearing a chimeric antigen receptor against prostate-specific membrane antigen mediate vascular disruption and result in tumor regression. Cancer Immunol Res. 2015; 3(1):68-84.

2. Risk M, Corman JM. The role of immunotherapy in prostate cancer: an overview of current approaches in development. Rev Urol. 2009; 11(1):16-27.

3. Shore N, Mason M, de Reijke TM. New developments in castrateresistant prostate cancer. BJU Int. 2012;109(Suppl 6):22-32.

4. Sanchez C, Chan R, Bajgain P, et al. Combining T-cell immunotherapy and anti-androgen therapy for prostate cancer. Prostate Cancer Prostatic Dis. 2013;16(2):123-131.

5. Abate-Daga D, Lagisetty KH, Tran E, et al. A novel chimeric antigen receptor against prostate stem cell antigen mediates tumor destruction in a humanized mouse model of pancreatic cancer. Hum Gene Ther. 2014;25(12):1003-1012.

6. Gill S, June CH. Going viral: chimeric antigen receptor T-cell therapy for hematological malignancies. Immunol Rev. 2015;263(1):68-89.

7. Kloss CC, Lee J, Zhang A, et al. Dominant-negative TGF- $\beta$ receptor enhances PSMA-targeted human CAR T cell proliferation and augments prostate cancer eradication. Mol Ther. 2018;26(7):1855-1866.

8. Duong CP, Westwood JA, Berry LJ, Darcy PK, Kershaw MH. Enhancing the specificity of T-cell cultures for adoptive immunotherapy of cancer. Immunotherapy. 2011;3(1):33-48.

9. Ramos CA, Dotti G. Chimeric antigen receptor (CAR)-engineered lymphocytes for cancer therapy. Expert Opin Biol Ther. 2011;11(7):855-873.

10. Kershaw MH, Westwood JA, Parker LL, et al. A phase I study on adoptive immunotherapy using gene-modified $\mathrm{T}$ cells for ovarian cancer. Clin Cancer Res. 2006;12(20 Pt1):6106-6115.

11. Carpenito C, Milone MC, Hassan R, et al. Control of large, established tumor xenografts with genetically retargeted human T cells containing CD28 and CD137 domains. Proc Natl Acad Sci U S A. 2009;106(9): 3360-3365.

12. Chmielewski M, Kopecky C, Hombach AA, Abken H. IL-12 release by engineered $\mathrm{T}$ cells expressing chimeric antigen receptors can effectively Muster an antigen-independent macrophage response on tumor cells that have shut down tumor antigen expression. Cancer Res. 2011; 71(17):5697-5706.

13. Mikolajczyk SD, Rittenhouse HG. Pro PSA: a more cancer specific form of prostate specific antigen for the early detection of prostate cancer. Keio J Med. 2003;52(2):86-91. 
14. Jöbsis AC, de Vries GP, Meijer AE, Ploem JS. The immunohistochemical detection of prostatic acid phosphatase: its possibilities and limitations in tumour histochemistry. Histochem J. 1981;13(6):961-973.

15. Sokoloff RL, Norton KC, Gasior CL, Marker KM, Grauer LS. A dualmonoclonal sandwich assay for prostate-specific membrane antigen: levels in tissues, seminal fluid and urine. Prostate. 2000;43(2):150-157.

16. Rajasekaran SA, Christiansen JJ, Schmid I, et al. Prostate-specific membrane antigen associates with anaphase-promoting complex and induces chromosomal instability. Mol Cancer Ther. 2008;7(7): 2142-2151.

17. Reiter RE, Gu Z, Watabe T, et al. Prostate stem cell antigen: a cell surface marker overexpressed in prostate cancer. Proc Natl Acad Sci US A. 1998;95(4):1735-1740.

18. Gu Z, Thomas G, Yamashiro J, et al. Prostate stem cell antigen (PSCA) expression increases with high gleason score, advanced stage and bone metastasis in prostate cancer. Oncogene. 2000;19(10):1288-1296.

19. Dannull J, Diener PA, Prikler L, et al. Prostate stem cell antigen is a promising candidate for immunotherapy of advanced prostate cancer. Cancer Res. 2000;60(19):5522-5528.

20. Ma Q, Gomes EM, Lo AS, Junghans RP. Advanced generation antiprostate specific membrane antigen designer $\mathrm{T}$ cells for prostate cancer immunotherapy. Prostate. 2014;74(3):286-296.

21. Kloss CC, Condomines M, Cartellieri M, Bachmann M, Sadelain M. Combinatorial antigen recognition with balanced signaling promotes selective tumor eradication by engineered T cells. Nat Biotechnol. 2013; 31(1):71-75
22. Priceman SJ, Gerdts EA, Tilakawardane D, et al. Co-stimulatory signaling determines tumor antigen sensitivity and persistence of CAR T cells targeting PSCA+ metastatic prostate cancer. Oncoimmunology. 2018;7(2):e1380764.

23. Zhang Q, Helfand BT, Carneiro BA, et al. Efficacy against human prostate cancer by prostate-specific membrane antigen-specific, transforming growth factor- $\beta$ Insensitive genetically targeted $\mathrm{CD} 8^{+}$ T-cells derived from patients with metastatic castrate-resistant disease. Eur Urol. 2018;73(5):648-652.

24. Singh S, Asal R, Bhagat S. Multifunctional antioxidant nanoliposomemediated delivery of PTEN plasmids restore the expression of tumor suppressor protein and induce apoptosis in prostate cancer cells. J Biomed Mater Res A. 2018;106(12):3152-3164.

25. Jakobczyk H, Sciortino F, Chevance S, Gauffre F, Troadec MB. Promises and limitations of nanoparticles in the era of cell therapy: example with CD19-targeting chimeric antigen receptor (CAR)modified T cells. Int J Pharm. 2017;532(2):813-824.

26. Soltanian S, Riahirad H, Pabarja A, Reza Karimzadeh M, Saeidi K. Kaempferol and docetaxel diminish side population and down-regulate some cancer stem cell markers in breast cancer cell line MCF-7. Biocell. 2017;41(2-3):33-40.

27. Hillerdal V, Essand M. Chimeric antigen receptor-engineered T cells for the treatment of metastatic prostate cancer. Bio Drugs. 2015;29(2): 75-89
OncoTargets and Therapy

\section{Publish your work in this journal}

OncoTargets and Therapy is an international, peer-reviewed, open access journal focusing on the pathological basis of all cancers, potential targets for therapy and treatment protocols employed to improve the management of cancer patients. The journal also focuses on the impact of management programs and new therapeutic agents and protocols on

\section{Dovepress}

patient perspectives such as quality of life, adherence and satisfaction. The manuscript management system is completely online and includes a very quick and fair peer-review system, which is all easy to use. Visit http://www.dovepress.com/testimonials.php to read real quotes from published authors. 